\title{
comportement des barrages en terre à noyau non saturé
}

\author{
R. SINNINGER \\ Professeur, Institut des Travaux Hydrauliques* \\ C.C.H. - Chaire de Constructions Hydrauliques \\ E. SEKER \\ Docteur-Ingénieur E.P.F.L., Insätut des Sols, Roches et Fondations*
}

\section{INTRODUCTION}

Pour les barrages en terre avec noyau central en matériau meuble cohérent, incliné ou non, les caractéristiques de cette zone peu perméable jouent un rôle important sous plusieurs aspects.

Lors de la construction, le matériau change constamment de propriétés, tout d'abord à la suite du compactage même, mais également comme conséquence du changement de l'état de contraintes du à l'augmentation couche par couche du remblai. Ainsi la résistance au cisaillement, la déformabilité et la perméabilité se développent individuellement en chaque point du noyau au cours de la construction pour arriver à un premier stade particulier pour le jugement de la stabilité, celui de la fin de la construction.

Il importe de souligner qu'à ce stade le matériau du noyau n'a donc pas partout les mêmes caractéristiques et qu'il est, le plus souvent, dans un état de non-saturation, au moins dans la partie supérieure du remblai.

Dans la phase d'exploitation le matériau du noyau continue, pour une période plus ou moins longue, de modifier ses caractéristiques, suite à la consolidation. Cette modification entraîne des déformations qui sont décisives pour la surélévation du couronnement et pour les ouvrages en contact avec le noyau. Ce phénomène de consolidation a de nouveau lieu le plus souvent dans un milieu non saturé. L'influence de l'eau de la retenue filtrant à travers le noyau est exclue des considérations suivantes. Ceci est d'autant plus justifié que l'état de saturation complète, si jamais atteint, nécessite une mise en eau de longue durée.

Dans la pratique courante et avec des modèles mathématiques plus ou moins sophistiqués, il est possible de calculer l'état de contrainte et les déformations pour n'importe quel point et à n'importe quel moment [1, 2]. Mais tous ces programmes, du moins à la connaissance des auteurs, ne tiennent pas explicitement compte de la succion matricielle qui est en étroite relation avec l'état de saturation d'un matériau cohérent. Ainsi les solutions numériques existantes ne tiennent pas compte des lois réelles qui gouvernent le comportement physique. En effet, l'interprétation actuelle des essais triaxiaux et le fait que la détermination de la succion matricielle n'appartienne pas encore aux essais courants de la classification, montrent que les phénomènes et les conséquences de la non-saturation sont, à l'heure actuelle, insuffisamment ou pas du tout

\footnotetext{
* Ecole Polytechnique Fédérale de Lausanne, CH - 1015 Lausanne, Suisse,
} 
considérés. Il n'est donc pas à exclure que des valeurs erronées des pressions interstitielles soient introduites dans les calculs ou que les pressions observées dans un noyau pendant et après la construction ne puissent pas être interprétées correctement.

La méthode de calcul proposée tient compte de la non-saturation du matériau lors de sa construction et permet, par un premier programme développé, la détermination des déformations instantanées lors de la mise en place des remblais. Un deuxième programme calcule les déformations différées d'un matériau non saturé, Leur combinaison reproduit fidèlement la construction d'un barrage en terre et fournit l'état de contraintes, pendant et après la réalisation. Il va de soi que ces informations sont indispensables pour l'analyse de la stabilité.

Pour une première estimation du comportement de la digue, sans recours aux programmes, des méthodes simplifiées pour les tassements instantanées et différés sont expliquées.

Finalement les programmes complets et la méthode simplifiée sont appliqués à un cas réel de barrage en terre. Leurs résultats sont comparés aux mesures d'auscultation.

\section{CONSIDÉRATIONS THÉORIQUES}

Pour calculer le comportement d'un sol non saturé, il faut considérer trois phénomènes élémentaires de la mécanique des sols, à savoir la déformabilité, l'écoulement du fluide composé d'eau et d'air et finalement la résistance. Les lois décrivant ces phénomènes sont sommairement développées ci-dessous en utilisant des formes matricielles pour les équations, afin de tenir compte de la nature tridimensionnelle du problème posé.

\subsection{Déformabilitê des sols non saturés}

La déformabilité du squelette minéral peut être décrite à l'aide du module d'élasticité $\mathrm{E}$ et du coefficient de Poisson $v$, celle du fluide par le coefficient de compressibilité $\mathrm{m}_{\mathrm{w}}$.

A la suite de l'application d'une charge, la déformation se manifeste en deux étapes bien distinctes:

- comportement à court terme: il en résulte la déformation instantanée, accompagnée d'une augmentation des pressions interstitielles;

- comportement à long terme: il en résulte la déformation différée qui évolue dans le temps, suite à la dissipation des pressions interstitielles sous des gradients variables.

Les déformations du milieu dans les deux étapes entraînent une modification des contraintes, de la porosité, de la perméabilité et de la saturation.

Ces deux comportements sont traités séparément ciaprès.

\subsubsection{Déformation instantanée}

La déformation instantanée se produit lors de l'application de la charge au temps $\mathrm{t}=0$. Elle est due à la compression de l'air d'une part et la dissolution d'une partie de l'air dans l'eau d'autre part. Une diminution du volume total en résulte.

A cause de la modification non linéaire des paramètres essentiels pour le comportement lors de la déformation instantanée un calcul incrémental est indispensable.

Il est admis que le milieu obéit à la loi de contraintesdéformations

$$
\{\Delta \varepsilon\}=[D]^{-1}\left\{\Delta \sigma^{\prime}\right\}
$$

où $\{\Delta \varepsilon\}=\left[\Delta \varepsilon_{1}, \Delta \varepsilon_{2}, \Delta \varepsilon_{3}\right]^{\mathrm{T}}$ décrit les déformations, $\left\{\Delta \sigma^{\prime}\right\}=\left[\Delta \sigma_{1}{ }^{\prime}, \Delta \sigma_{2}{ }^{\prime}, \Delta \varepsilon_{3}{ }^{\prime}\right]^{\mathrm{T}}$ les contraintes effectives et [D] la matrice d'élasticité. Cette dernière prend la forme:

$$
[D]=E_{\text {oed }}\left[\begin{array}{rrr}
1 \frac{v}{1-v} & \frac{v}{1-v} \\
\text { sym } & 1 & \frac{v}{1-v} \\
& 1
\end{array}\right]
$$

Le module œedométrique $\mathrm{E}_{\text {oed }}$ peut être défini à l'aide d'une loi exponentielle de la forme:

$$
E_{\mathrm{oed}}=\mathrm{m}_{0}\left(\frac{\sigma_{1}^{\prime}}{\mathrm{p}_{\mathrm{a}}}\right)^{\mathrm{m}_{1}}
$$

d'où le module d'élasticité $E=E_{\text {oed }}[(1+v)$

$(1-2 v) /(1-v)]$.

$m_{0}$ et $m_{1}$ sont des paramètres qui peuvent être définis à l'aide d'essais œdométriques, $\sigma_{1}$ ' est la contrainte effective appliqué et $\mathrm{p}_{\mathrm{a}}$ la pression atmosphérique $\left[\simeq 100 \mathrm{kN} \cdot \mathrm{m}^{-2}\right]$ (fig. 1)

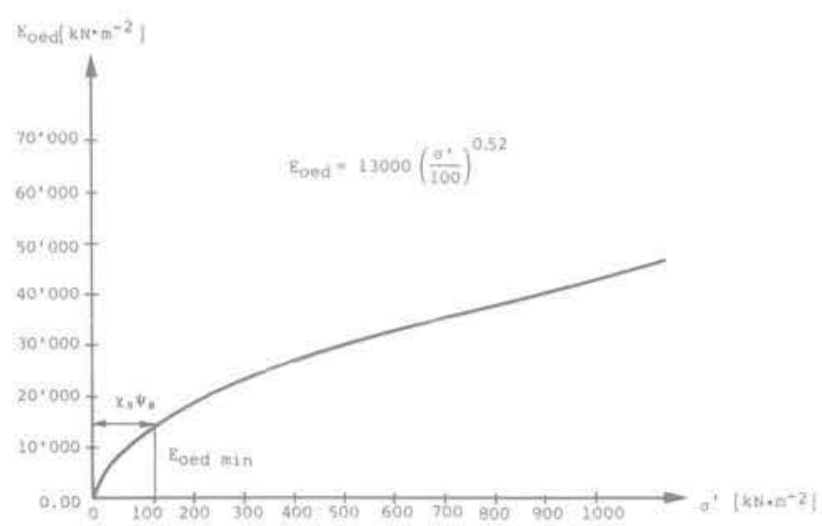

Fig. 1. - Module d'élasticité $E_{\text {oed }}$ en fonction de $\sigma^{\prime}$.

La variation de la contrainte effective $\Delta \sigma^{\prime}$ dans 1 peut s'exprimer par la formule:

$$
\left\{\Delta \sigma^{\prime}\right\}=\{\Delta \sigma\}-\{\Delta \mathrm{p}\}
$$


dans laquelle $\Delta \sigma$ signifie la variation de la contrainte totale et $\Delta p$ la variation de la pression interstitielle (air + eau). Sous l'hypothèse de la validité de la loi de Skempton $\Delta \mathrm{p}=\mathrm{B}\left[\Delta \sigma_{3}+\mathrm{A}\left(\Delta \sigma_{1}-\Delta \sigma_{3}\right)\right]$, cette dernière s'écrit sous la forme matricielle:

$$
\{\Delta \mathrm{p}\}=[\mathrm{U}]\{\Delta \sigma\} \text {, }
$$

La matrice de pression interstitielle [U] ne dépend que des coefficients $A$ et $B$ de la loi de Skempton:

$$
[U]=\left[\begin{array}{ll}
A B & B-A B \\
A B & B-A B
\end{array}\right]
$$

En introduisant les équations 3 et 4 dans 1 , on obtient les déformations en fonction des contraintes totales $\Delta \sigma$ et des pressions interstitielles caractérisées par $A$ et $B$ :

$$
\{\Delta \varepsilon\}=\left[[D]^{-1}-[D]^{-1}[U]\right]\{\Delta \sigma\} \text {. }
$$

En posant:

$$
\left[D_{u}\right]^{-1}=\left[[D]^{-1}-[D]^{-1}[U]\right],
$$

on obtient la matrice d'élasticité d'un milieu non saturé et non drainé qui tient compte séparément des caractéristiques de chacune des trois phases. Elle s'écrit

$$
\{\Delta \varepsilon\}=\left[D_{u}\right]^{-1}\{\Delta \sigma\}
$$

avec

$$
\left[D_{u}\right]=\frac{E_{u}\left(1-v_{u}\right)}{\left(1+v_{u}\right)\left(1-2 v_{u}\right)}\left[\begin{array}{rr}
1 \frac{v_{u}}{1-v_{u}} & \frac{v_{u}}{1-v_{u}} \\
\text { sym } & 1 \frac{v_{u}}{1-v_{u}} \\
1
\end{array}\right] \text { (8) }
$$

Dans cette expression $E_{u}=E /[1-A B(1-2 v)]$ est le modèle d'élasticité non drainé et $v_{u}=0.5[1-$ $(1-B)(1-2 v) /[1-A B(1-2 v)]]$ correspond au coefficient de Poisson non drainé.

Pour chiffrer ces deux valeurs caractérisant les déformations du milieu non saturé et non drainé, les coefficients $\mathrm{A}$ et $\mathrm{B}$ doivent être déterminés. Notons que A se rapporte au milieu et $B$ au milieu et au fluide. Les deux sont des variables, puisque le milieu et le fluide changent pendant la déformation.

L'expression de A est une approximation, basée sur l'hypothèse que pour $\sigma_{1} \leqslant \sigma_{3} / k o$, le milieu est considéré comme isotrope et élastique. Pour $\sigma_{1}>\sigma_{3} / k_{0}$ l'évolution de $\mathrm{A}$ en fonction du déviateur est indiquée dans la formule suivante (fig. 2):

$A=\frac{\left(A_{f}-1 / 3\right)}{\left(\Delta q_{f}-\Delta q_{k o}\right)}\left(\Delta q-\Delta q_{k o}\right)+1 / 3$.

$A_{f}$ est le coefficient $A$ au moment de la rupture. S'il n'existe pas d'essais, $A_{f}$ peut être estimé à l'aide de l'expression simplifiée suivante qui se trouve confirmée par les essais de Henkel [3]:

$$
A_{\mathrm{f}}=1 / 3\left(\frac{4}{\mathrm{OCR}}-1\right)
$$

$\Delta \mathrm{q}_{\mathrm{f}}=\left(\sigma_{1}-\sigma_{3}\right)_{\mathrm{f}}$ correspond au déviateur à la rupture et $\Delta \mathrm{q}_{\mathrm{ko}}=\left(\sigma_{1}-\sigma_{3}\right)_{\mathrm{ko}}$ correspond au déviateur pour le rapport des contraintes à l'état de repos, donc

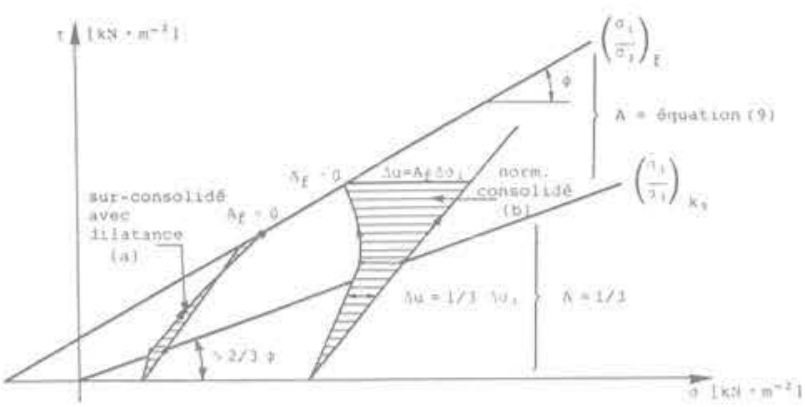

Fig. 2. - Augmentation de la pression interstitielle et calcul du coefficient $A$ pour les cas d'un sol sur-consolidé (a) et normalement consolidé (b) pour $B=1$.

$\sigma_{1} / \sigma_{3}=$ ko $=v /(1-v)$ ou encore, selon Jacky, $\sigma_{1} / \sigma_{3}=1-\sin \phi$. OCR caractérise le degré de surconsolidation et résulte du rapport $\sigma_{c} / \sigma_{v}$, dans lequel $\sigma_{c}$ est égal à la contrainte de consolidation et $\sigma_{v}$ décrit l'état de contrainte totale actuel.

Le calcul de $\mathrm{B}$ nécessite le développement suivant. Dans le cas d'un sol non saturé, les pores sont remplis d'air et d'eau. La pression interstitielle totale $p$ se compose d'une pression $u_{\mathrm{a}}$ transmise par l'air et d'une pression $u_{w}$ transmise par l'eau. En introduisant ces deux pressions séparément, Bishop [4] a défini la contrainte effective $\sigma^{\prime}=\sigma-p$ de la manière suivante:

$$
\sigma^{\prime}=\sigma-\left[u_{a}-\chi\left(u_{a}-u_{w}\right)\right] .
$$

$\chi$ appelé le coefficient de Bishop dépend du degré de saturation $\mathrm{S}_{\mathrm{r}}$ et s'écrit:

$$
\chi=\left(\frac{\mathrm{S}_{\mathrm{r}}-\chi_{1}}{1-\chi_{1}}\right)^{\gamma_{2}}
$$

Les deux paramètres $\chi_{1}$ et $\chi_{2}$ caractérisent le sol (fig. 3). En outre, la différence des pressions $\left(u_{a}-u_{w}\right)=\psi$ est appelée succion matricielle, dépendant également du degré de saturation $\mathrm{S}_{\mathrm{r}}$ (fig. 4).

B n'est donc plus une constante et s'écrit:

$$
\mathrm{B}=\frac{\Delta u_{\mathrm{a}}-\Delta(\chi \psi)}{\Delta \sigma}
$$

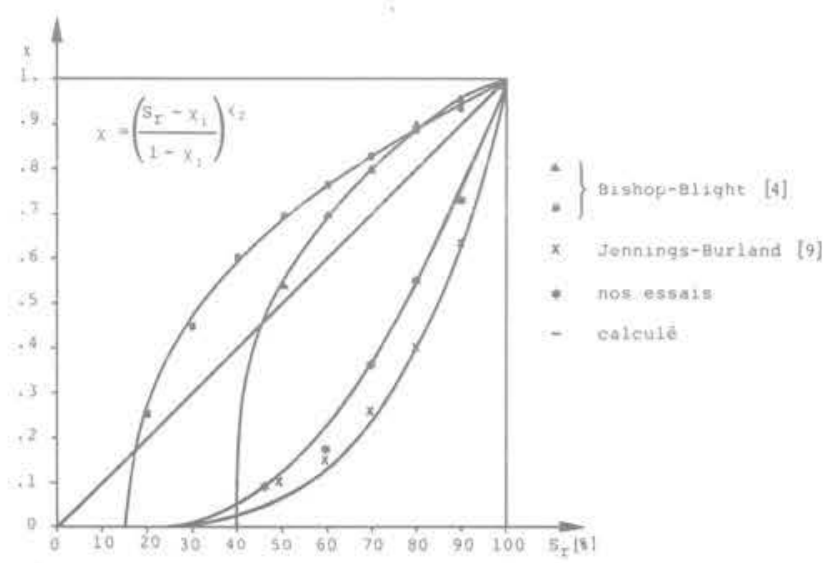

Fig. 3. - Coefficient $\chi$ de Bishop en fonction de $S_{r}$ pour différents types de sol. 


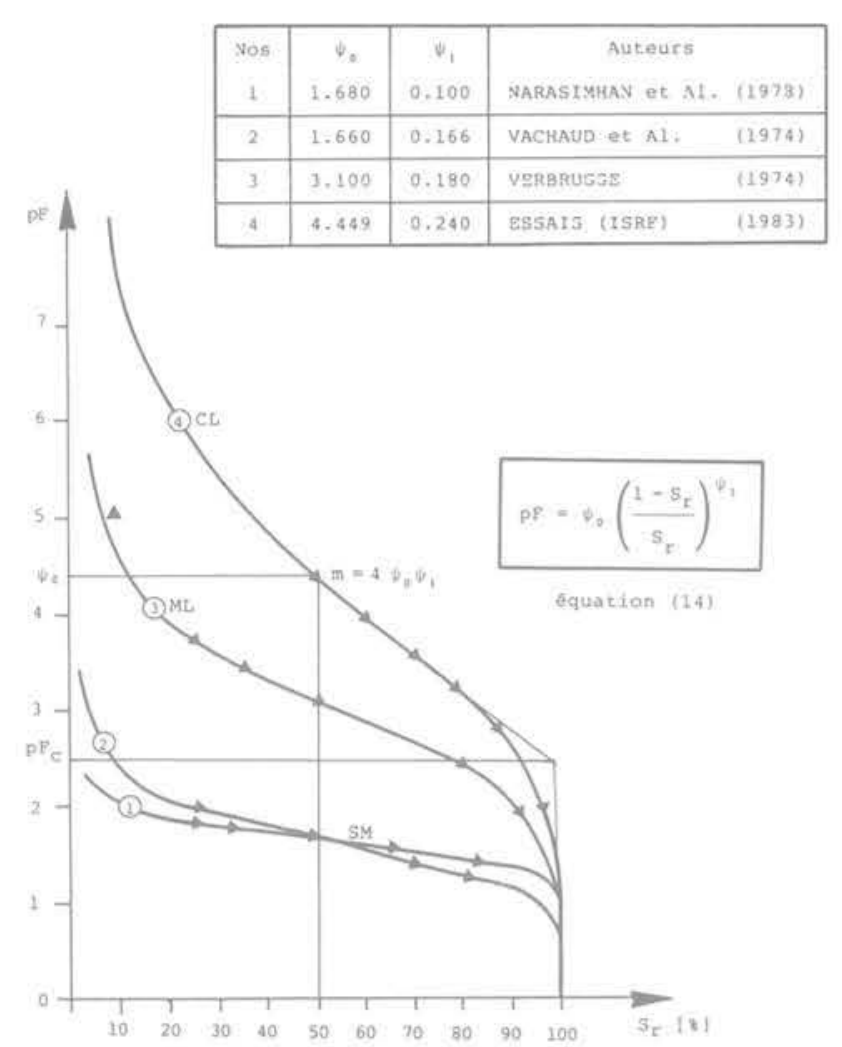

Fig. 4. - pF en fonction de $S_{r}$ avec $\boldsymbol{\Delta}$ pour les valeurs moyennes mesurées.

où $\Delta u_{a}$ est l'augmentation de la pression de l'air lors de l'augmentation de la contrainte totale $\Delta \sigma, \chi$ étant le coefficient de Bishop et $\psi$ la succion matricielle. Cette dernière, caractérisant l'interaction des trois phases dans un sol non saturé, est d'une importance primordiale sur l'évolution des pressions interstitielles. Par définition :

$$
\psi=\left(u_{a}-u_{w}\right)=\gamma_{w} \cdot 10^{p F} .
$$

L'exposant $\mathrm{pF}$ est fonction de la saturation $\mathrm{S}_{\mathrm{r}}$ d'une part et de la nature du sol d'autre part, comme illustré dans la figure 4. Sa forme analytique s'écrit:

$$
\mathrm{pF}=\psi_{0}\left(\frac{1-S_{r}}{S_{r}}\right)^{\psi_{1}}
$$

Les paramètres $\psi_{0}$ et $\psi_{1}$ traduisent la nature du sol. Il a été démontré [5] que la pente $\mathrm{m}$ de la courbe $\mathrm{pF}\left(\mathrm{S}_{\mathrm{r}}\right)$ pour une saturation $\mathrm{S}_{\mathrm{r}}=50 \%$ peut s'exprimer $\mathrm{m}=-4 \psi_{0} \psi_{1}$. En considérant que $\psi_{0}=\mathrm{pF}$ pour $\mathrm{S}_{\mathrm{r}}=50 \%$ et en connaissant $\mathrm{m}$ pour la même saturation, les paramètres $\psi_{0}$ et $\psi_{1}$ peuvent être calculés.

Le coefficient de pression interstitielle $B$ en fonction de la même saturation $\mathrm{S}_{\mathrm{r}}$ peut être calculé à l'aide de l'équation (13), comme démontré plus loin dans l'exemple numérique.

Les caractéristiques du milieu $E_{u}$ et $v_{u}$ sont ainsi connues et l'équation (5) permet le calcul des déformations $\Delta \varepsilon$.

\subsubsection{Déformation différée}

Après la déformation instantanée, le sol continue de se déformer sous l'effet de la dissipation des pressions interstitielles. Cette déformation, dite déformation différée, évolue au cours du temps. Si l'on suppose que seule l'eau s'écoule à travers le milieu poreux, l'équation de continuité s'écrit:

$$
\operatorname{div}\left(v_{w}\right)=m_{a} m_{v c} \frac{\partial \sigma_{\text {oct }}^{\prime}}{\partial t}
$$

où :

$$
\mathrm{m}_{\mathrm{a}}=\mathrm{s} \cdot \mathrm{n}(1-\mathrm{n})+\mathrm{S}_{\mathrm{r}}
$$

est le paramètre de la compressibilité et

$$
s=\partial S_{r} / \partial \psi \cdot \partial \psi / \partial n
$$

est appelé la capacité spécifique de saturation avec $\psi$ la succion matricielle, $\mathrm{S}_{\mathrm{r}}$ la saturation et $\mathrm{n}$ la porosité; $\sigma_{\text {oct }}^{\prime}$ est le tenseur sphérique.

Pour l'écoulement à l'intérieur d'un milieu non saturé en cours de consolidation, il est admis que la loi généralisée de Darcy est valable. L'air se trouve donc discontinu (occlus) dans l'eau sous forme de bulles qui restent attachées aux grains. Sous ces conditions seule l'eau s'écoule à travers le milieu. Il faut pourtant préciser que, selon les conditions de contraintes et de pressions, l'air se dissout plus ou moins dans l'eau en obéissant aux lois de Mariotte et de Henry.

La loi de Darcy généralisée s'écrit sous forme matricielle pour un milieu non saturé:

$$
\{\mathrm{v}\}=-[\mathrm{k}] \nabla\{\mathrm{h}\}
$$

avec $\{v\}$ le vecteur de la vitesse moyenne d'écoulement, [k] la matrice de perméabilité du milieu et $\{\mathrm{h}\}$ le vecteur du gradient hydraulique. Dans le cas de la non saturation, le coefficient de perméabilité devient:

$$
\mathrm{k}=\mathrm{k}_{\mathrm{wr}} \cdot \mathrm{k}_{\mathrm{w}}
$$

où $k_{w}$ est la perméabilité à l'eau d'un sol saturé. La perméabilité relative

$$
k_{w r}=\left(\frac{S_{r}-S_{r \min }}{1-S_{r \min }}\right)^{n_{1}}
$$

tient compte, en plus de la saturation $\mathrm{S}_{\mathrm{r}}$, de la saturation minimale $S_{r} \min$ et d'un paramètre $n_{1}$ caractérisant le milieu.

\subsection{Résistance}

La résistance du squelette minéral définie par l'angle de frottement $\phi$ et la cohésion $c$, est caractérisée par l'existence d'un seuil de sollicitation au-dessus duquel apparaissent des déformations permanentes importantes. Pour tenir compte de ce fait, il faut définir deux lois différentes de comportement:

- une loi de rupture;

- une loi d'écoulement plastique.

Le critère Mohr-Coulomb pour la loi de rupture (fig. 5) et une loi d'écoulement élasto-parfaitement plastique avec une loi associée (fig. 6) ont été choisis. En calcul incrémental, par la méthode des éléments finis, il en 


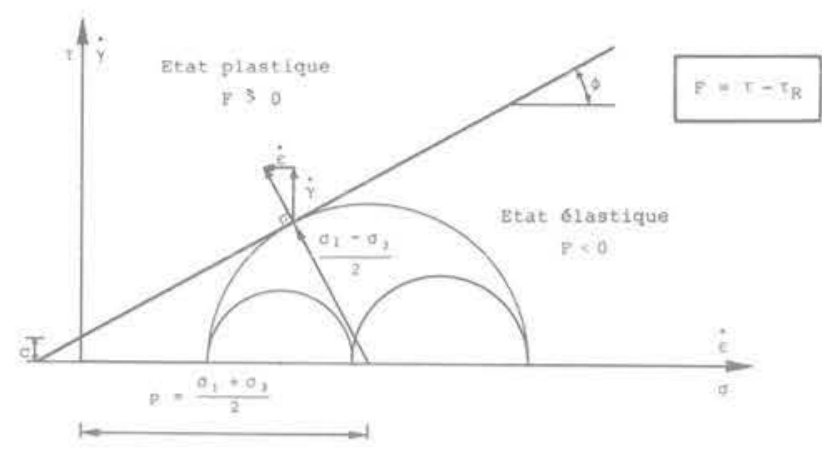

Fig. 5. - Loi de Mohr-Coulomb.

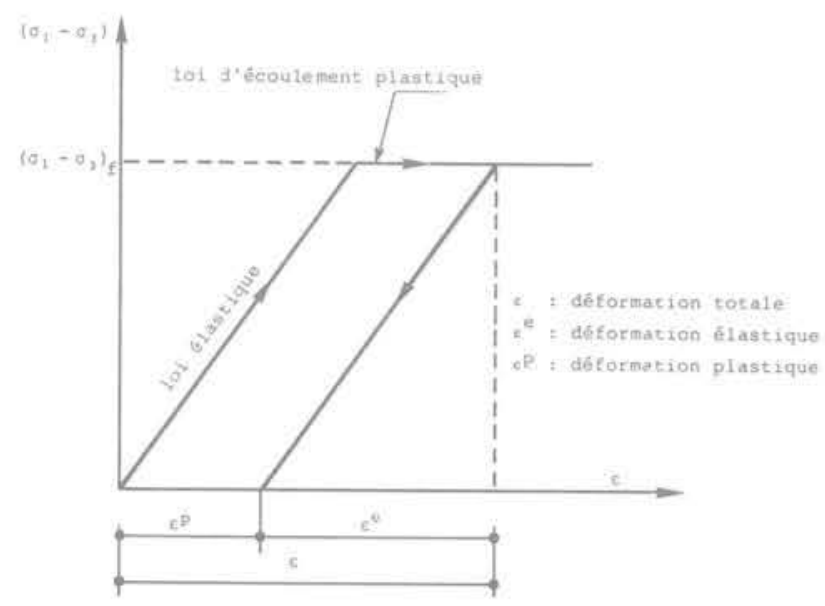

Fig. 6. - Comportement élasto-parfaitement plastique.

résulte une matrice de rigidité variable. Le système est résolu en modifiant la matrice à chaque itération en fonction des contraintes qui ont été calculées au cours de l'itération précédente [5].

\section{DÉTERMINATION DES CARACTÉRISTI- QUES D'UN MILIEU NON SATURÉ}

\subsection{Succion}

La courbe $\mathrm{pF}-\mathrm{S}_{\mathrm{r}}$ d'un sol (fig. 4) s'établit en laboratoire par des essais particuliers. La technique la plus utilisée soumet l'échantillon à des pressions variables en lui permettant en même temps de s'essorer. Ainsi à chaque pression, exprimée en log de la colonne d'eau en $\mathrm{cm}(\mathrm{pF})$, se détermine un degré de saturation $S_{r}$. De l'équation 15 on tire $\psi_{0}$ et $\psi_{1}$. Finalement $\psi$ est calculé à l'aide de l'équation 14 . Seker a démontré [5] que pF ainsi que $\psi_{0}$ et $\psi_{1}$ peuvent être estimés à l'aíde de l'indice de plasticité $I_{p}$ et de la limite de liquidité $w_{\mathrm{L}}$.

\subsection{Coefficient de Bishop}

Le coefficient de Bishop $\chi$ peut être déterminé par des essais triaxiaux sur des échantillons non saturés.
Connaissant les valeurs $\phi$ ' et ' c' ainsi que les contraintes totales au moment de la rupture on peut en déduire $\Delta p$ et l'équation 11 donne $\chi=\left(u_{a}-\Delta p\right) / \psi$. Dans un essai de compression simple $u_{a}$ peut être négligé et $\chi=\sigma_{3}{ }^{\prime} / \psi$ vu que $\sigma_{3}{ }^{\prime}=-\Delta \mathrm{p}$.

S'il s'agit d'une première estimation de $\chi$ au moment où les essais triaxiaux font encore défaut, l'équation 12 peut être utilisée en posant $\chi_{1}=0.25$ (sols cohérents) et $\chi_{2}=2 \log \psi_{0}+1[5]$.

\subsection{Perméabilité relative à l'eau}

Les méthodes utilisées pour déterminer la perméabilité relative à l'eau $k_{w r}$ sont basées sur les mesures de la saturation et de la succion [6]. L'équation 21 peut être résolue en posant $S_{r \min }=0.25$ et en exprimant $n_{1}$ en fonction du paramètre de succion $\psi_{0}$ de la façon suivante: $n_{1}=2 \log \psi_{0}+3[5]$.

\section{CALCUL SIMPLIFIÉ}

Si l'on considère un cas unidimensionnel et un degré de saturation correspondant à une teneur en eau proche de l'optimum Proctor, une méthode simplifiée peut être proposée.

Le tassement instantané dans un milieu non drainé se calcule à partir des conditions initiales en utilisant la notion de contraintes effectives et en introduisant la succion.

Les tassements différés sont calculés selon la méthode de Terzaghi en introduisant un coefficient de correction pour tenir compte du degré de saturation et de la succion.

La méthode simplifiée permet, dans une première approche et sans recourir aux programmes d'ordinateur, une rapide estimation des contraintes, tassements et variations des pressions interstitielles.

\subsection{Tassement instantanē}

Dans un premier pas il faut définir la courbe des déformations verticales $\varepsilon_{y}$ en fonction des contraintes effectives verticales $\sigma_{v}$ ' à l'aide d'essais œdométriques ou, en leur absence, par la formule 2.

La figure 7 montre une telle courbe dont l'expression analytique s'écrit:

$$
\Delta \varepsilon_{\mathrm{v}}=\frac{\Delta \sigma_{\mathrm{v}}{ }^{\prime}}{\mathrm{E}_{\mathrm{oed}}}
$$

Rappelons que la courbe œdométrique est basée sur un coefficient de poussée des terres au repos ko $=$ $v /(1-v)=1-\sin \phi^{\prime}$. Pour d'autres valeurs du coefficient de poussée des terres $k$, la courbe se calcule de la manière suivante:

$\Delta \varepsilon_{v}=\frac{\Delta \sigma_{v}^{\prime}}{E_{\text {oed }}} \cdot \frac{[1-v(1+k)](1-v)}{(1-2 v)}$ 


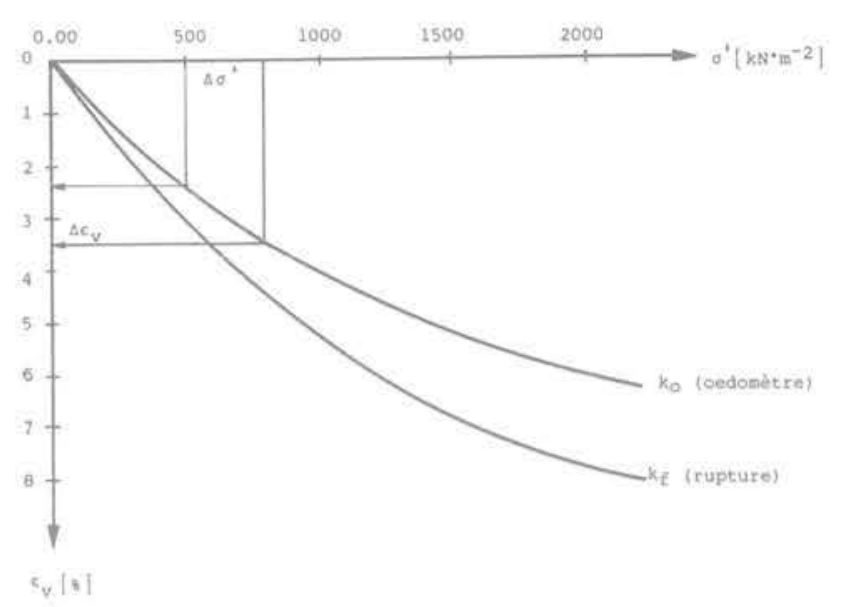

Fig. 7. - Courbe $\varepsilon_{v}-\sigma^{\prime}$.

La figure 7 montre une deuxième courbe pour laquelle le coefficient de la poussée correspond à celui de la rupture $k_{f}=\left(1-\sin \phi^{\prime}\right) /\left(1+\sin \phi^{\prime}\right)$. Au centre d'un remblai la déformation $\Delta \varepsilon\left(\Delta \sigma^{\prime}\right)$ est proche de la courbe pour $k_{0}$, tandis que vers les bords elle se déplace en direction de la courbe $k_{f}$.

Sous l'effet d'une augmentation $\Delta \sigma_{v}$ de la contrainte verticale totale, la pression interstitielle équivalente augmente de $\Delta \mathrm{p}$. Les contraintes totales $\Delta \sigma_{v}$ sont donc liées aux contraintes effectives $\Delta \sigma_{v}{ }^{\prime}$ par $\Delta \sigma_{v}{ }^{\prime}=$ $\Delta \sigma_{\mathrm{y}}-\Delta \mathrm{p}$. Le dernier terme se décompose en:

$$
\Delta \mathrm{p}=\Delta \mathrm{u}_{\mathrm{a}}-\Delta(\chi \psi)
$$

Le coefficient de Bishop $\chi$ est calculé selon l'équation 12 , tandis que la succion matricielle $\psi$, est obtenue à l'aide de l'équation 14. L'augmentation de la pression de l'air $\Delta u_{a}$ se calcule en appliquant la loi de Mariotte et celle de la dissolution des gaz dans l'eau de Henry (constante $\mathrm{H}=0.02$ ):

$$
\Delta u_{\mathrm{a}}=p a \frac{\varepsilon_{\mathrm{v}}}{e_{\mathrm{o}} \mathrm{D}-\varepsilon_{\mathrm{v}}} .
$$

$\mathrm{pa}=100 \mathrm{kN} \cdot \mathrm{m}^{-2}$ correspond à la pression atmosphérique, $e_{0}$ est l'indice de vide initial et $\mathrm{D}=$ $1-0.98 \mathrm{~S}_{\mathrm{ro}}, \mathrm{S}_{\mathrm{ro}}$ étant la saturation initiale. En calculant $\Delta \mathrm{u}_{\mathrm{a}}$ pour différents $\Delta \sigma_{v}^{\prime}$, une courbe $\mathrm{u}_{\mathrm{a}}\left(\sigma^{\prime}\right)$ peut être dessinée comme indiqué à la figure $8 \mathrm{~b}$.

Pour chaque augmentation de $\Delta \sigma_{v}^{\prime}$, le volume $n$ des pores diminue à cause de la compression. La teneur en eau restant constante (cas non drainé), la saturation $\mathrm{S}_{\mathrm{r}}$ augmente également:

$$
\mathrm{Sr}=\frac{\mathrm{S}_{\mathrm{ro}} \cdot \mathrm{n}_{\mathrm{O}}}{\mathrm{n}}=\frac{\mathrm{S}_{\mathrm{ro}} \cdot \mathrm{n}_{\mathrm{O}}}{\mathrm{n}_{\mathrm{0}}-\Delta \mathrm{n}}=\frac{\mathrm{S}_{\mathrm{ro}} \cdot \mathrm{n}_{\mathrm{0}}}{\mathrm{n}_{0}-\Delta \varepsilon_{\mathrm{v}}\left(1-\mathrm{n}_{0}\right)}
$$

$S_{r o}$ et $n_{0}$ sont respectivement la saturation et la porosité initiale et $\Delta \varepsilon_{v}=\Delta \sigma_{v}^{\prime} / E_{\text {oed. }}$. Partant de $S_{r o}$ et $n_{0}$, une courbe $S_{r}\left(\Delta \sigma^{\prime}\right)$ peut être calculée telle que représentée à la figure 9 .

Pour calculer $\psi\left(\sigma_{v}^{\prime}\right)$, on part des deux paramètres $\psi_{0}$ et $\psi_{1}$ connus, en introduisant dans la formule 14 pour chaque o' la saturation $S_{r}$ définie selon 28 .
Se basant sur $\psi=u_{a}-u_{w}$ on obtient $u_{w}\left(\sigma_{v}{ }^{\prime}\right)$, également reporté sur la figure $8 \mathrm{~b}$.

Le coefficient de Bishop $\chi\left(\sigma_{v}{ }^{\prime}\right)$ se calcule à partir de $\chi_{1}$ et $\chi_{2}$ selon (12). Le résultat est reporté sur la figure 9.

La connaissance de $u_{a}, \chi$ et $\psi$ en fonction de $\sigma_{v}$ ' permet, par l'intermédiaire de la définition de la pression interstitielle équivalente $\Delta p=\Delta u_{a}-\Delta(\chi \psi)$, le calcul de la fonction $\mathrm{p}\left(\sigma_{\mathrm{v}}\right)$. Dans la figure $8 \mathrm{c}, \mathrm{p}$ est reporté à l'abscisse $\sigma=\sigma_{\mathrm{v}}{ }^{\prime}+\mathrm{p}$.

Dans la pratique le rapport $\overline{\mathrm{B}}=\Delta \mathrm{p} / \Delta \sigma_{\mathrm{v}}$, avec $\Delta \sigma_{\mathrm{v}}=$ $\Delta \sigma_{\mathrm{v}}{ }^{\prime}+\Delta \mathrm{p}$, est souvent utilisé et indique dans quelle mesure la contrainte verticale totale $\sigma_{\mathrm{v}}$ est transmise au fluide interstitiel (air + eau).

Une fois les diagrammes des figures 8 et 9 élaborés, les opérations pour calculer $\Delta p$ et $\Delta \varepsilon$ d'une première augmentation de la contrainte totale $\Delta \sigma_{v_{1}}$ sont mentionnées ci-dessous:

(1) Reporter $\Delta \sigma_{\mathrm{v}_{1}}$ sur l'abscisse de la fig. $8 \mathrm{c}$ et définir $\Delta \mathrm{p}_{1}$.

(2) A l'aide de la définition $\Delta \sigma_{v}^{\prime}=\Delta \sigma_{v}-\Delta \mathrm{p}$ on détermine $\Delta \sigma_{v}^{\prime}$ et la figure $8 \mathrm{a}$ permet de trouver $\Delta \varepsilon_{1}$.

(3) La figure $8 \mathrm{~b}$ permet de déterminer $\Delta \mathrm{u}_{w}$.

La marche à suivre pour calculer une seconde augmentation de la contrainte $\Delta \sigma_{v_{2}}$ est également indiquée dans les figures. Notons qu'aucune dissipation de la pression interstitielle n'est considérée pour le tassement instantané.

\subsection{Tassements différés}

L'application d'une contrainte totale verticale $\Delta \sigma_{v}$ conduit à une augmentation de la pression interstitielle équivalente de $\Delta \mathrm{p}$. L'équation différentielle de la consolidation, sous condition $\Delta \sigma_{v}=c t e$, sol isotrope et phénomène unidimensionnel, s'écrit:

$$
c_{v} \frac{\partial^{2} \Delta p}{\partial_{z}^{2}}=\frac{\partial \Delta p}{\partial t}
$$

$c_{y}$ est le coefficient de consolidation qui, pour un milieu non saturé, prend la forme [5]

$$
c_{v}=\frac{K}{\gamma_{w} \cdot m_{a} \cdot m_{v c}}\left[m^{2} \cdot s_{1}^{-}\right]
$$

k a été défini par (20) et inclut la perméabilité relative $k_{w r}(\mathrm{Sr})$ décrit par (21). Le paramètre de compressibilité $\mathrm{m}_{\mathrm{a}}\left(\mathrm{S}_{\mathrm{r}}, \psi, \mathrm{n}\right)$ est donné par (17).

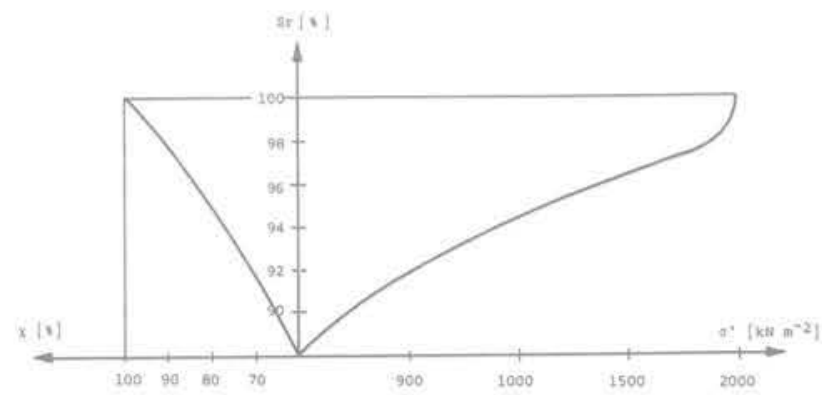

Fig. 9. $-S_{r}$ et $\chi$ en fonction de $\sigma_{v}^{\prime}$. 
En décomposant (28) en une première partie dite classique selon Terzaghi [8] (sol saturé) et une deuxième partie qui tient compte de la non saturation [10], on obtient:

$$
c_{v}=\frac{k_{w}}{\gamma_{w} \cdot m_{v c}} \cdot \frac{k_{w r}}{s \cdot n(1-n)+S_{r}}
$$

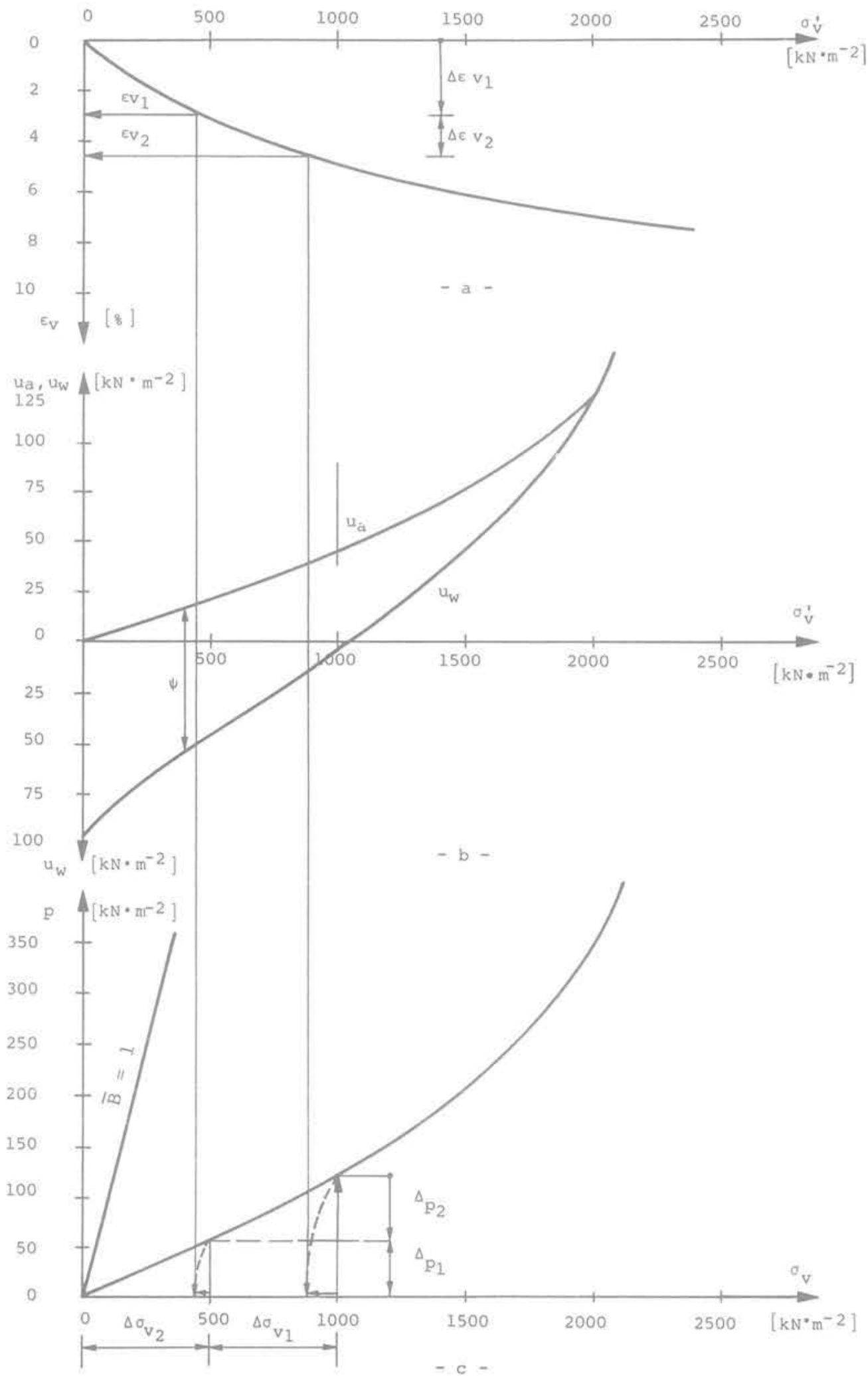

Fig. 8. $-\varepsilon_{v}, u_{a}, u_{w}$ et $p$ en fonction des contraintes. 
où avec $c_{\text {yo }}$ comme coefficient de consolidation dans le cas saturé et c comme coefficient de correction pour tenir compte de $S_{r}, \psi$ et $\mathrm{n}$ il en résulte:

$$
c_{v}=c_{v o} \cdot c
$$

avec:

$$
c=\frac{k_{w r}}{s \cdot n(1-n)+S_{r}}
$$

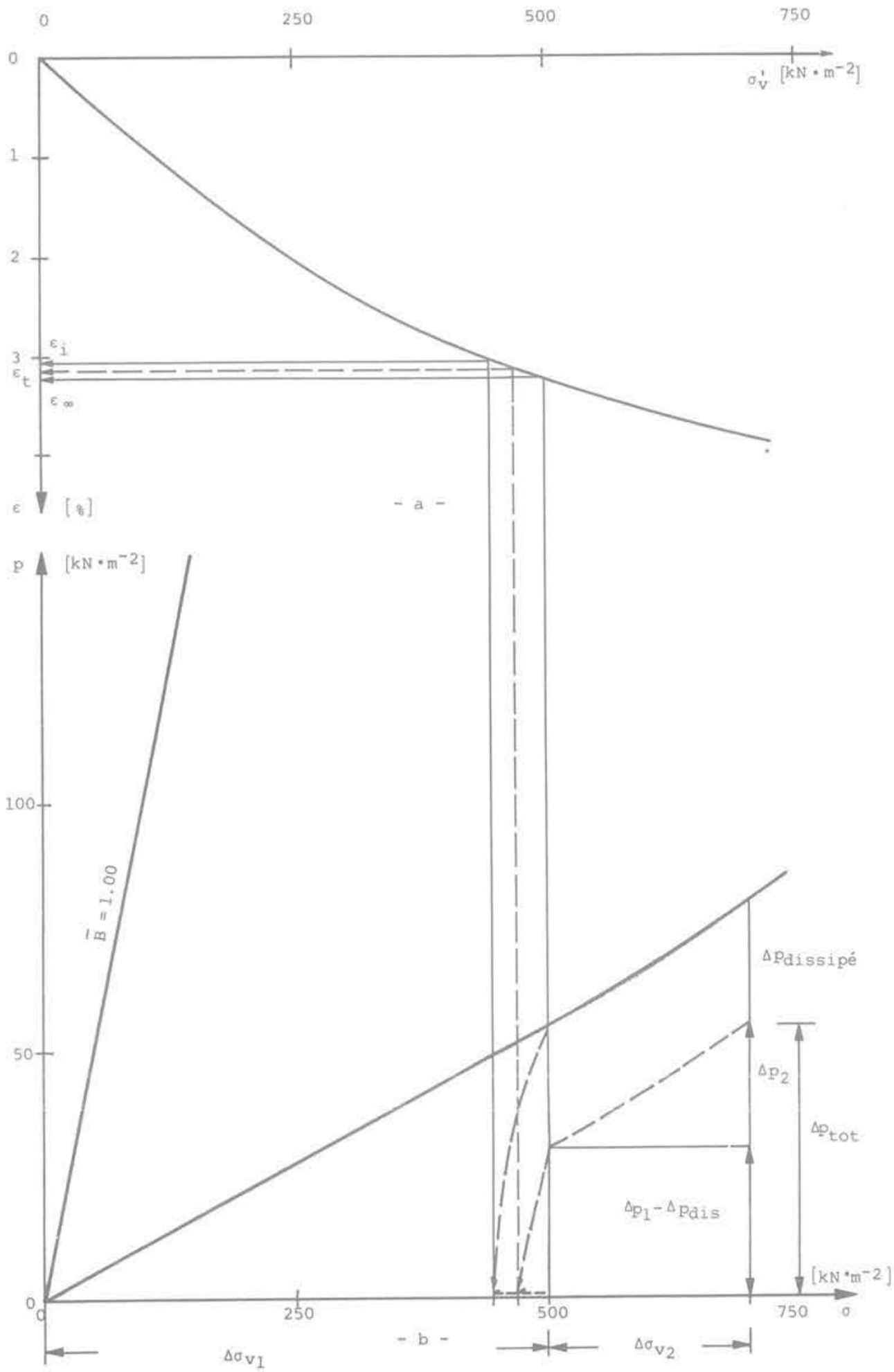

Fig. 10. $-\varepsilon_{v}$ et $p$ dissipé en fonction des contraintes. 
Pour calculer $c$ il faut donc disposer des valeurs $n_{1}$ relatives à la perméabilité selon (21) et s relative à la succion selon (18). Sa valeur est donnée dans l'annexe en fonction de la déformation volumétrique $\varepsilon_{\mathrm{v}}$, de la saturation $\mathrm{S}_{\mathrm{r}}$, de l'indice de plasticité $\mathrm{I}_{\mathrm{p}}$ et de la limite de liquidité $w_{\mathrm{L}}$.

Le degré de consolidation $\mathrm{U}_{\mathrm{ns}}$ dans un milieu non saturé est le rapport en \% des tassements $\Delta \mathrm{h}_{\mathrm{t}}$ au temps $t$ et des tassements $\Delta \mathrm{h}_{\infty}$ après dissipation complète des pressions interstitielles. $U$ est une fonction de facteur de temps $T_{v}$ bien connu, qui doit pourtant être complété pour le cas non saturé par le coefficient de correction c de l'équation 30b:

$$
T_{\text {vns }}=c T_{v}=c \frac{c_{v o} \cdot t}{H^{2}}
$$

$H$ est le chemin de percolation le plus court en un endroit donné et $\mathrm{t}$ le temps écoulé depuis l'application de la charge $\Delta \sigma$. La fonction $U\left(T_{v}\right)$ se trouve dans des abaques ou graphique de la littérature.

Le tassement différé au temps t pour un $c_{v}$ et un $\mathrm{H}$ donnés se détermine de la manière suivante (fig. 10):

(1) Calculer c selon $30 \mathrm{~b}$, ce qui permet de définir $\mathrm{T}_{\mathrm{vns}}$.

(2) D'un abaque donné dans la littérature on tire $U$, en fonction du $T_{\text {uns }}$ calculé.

(3) Sur la figure $10 \mathrm{~b}$ on reporte $\Delta \sigma$, ce qui permet la définition de $\Delta \mathrm{p}$ et, à l'aide de la figure $10 \mathrm{a}$ on trouve $\varepsilon_{i}$ à l'abscisse $(\Delta \sigma-\Delta p)$ et $\varepsilon_{\infty}$ à l'abscisse $\Delta \sigma$.

(4) La déformation $\varepsilon_{t}$ au temps $t$ est obtenue par

$$
\varepsilon_{\mathrm{t}}=\varepsilon_{\mathrm{i}}+\mathrm{U}\left(\varepsilon_{\infty}-\varepsilon_{\mathrm{i}}\right)
$$

(5) $\varepsilon_{\mathrm{t}}$ ainsi défini permet sur la figure $10 \mathrm{~b}$ de trouver la part de $\Delta \mathrm{p}$ dissipé.

Pour une deuxième augmentation de la charge totale $\Delta \sigma_{v_{2}}$ on définit à l'aide de la figure $10 \mathrm{~b}$ le $\Delta \mathrm{p}_{2}$ résultant. La pression équivalente totale, après l'application de la charge, doit tenir compte de la dissipation calculée au point (5)

$$
\Delta \mathrm{p}_{\mathrm{tot}}=\Delta \mathrm{p}_{1}-\Delta \mathrm{p}_{\text {dissipe }}+\Delta \mathrm{p}_{2}
$$

\section{CALCUL D'UN BARRAGE EN TERRE}

La théorie développée dans le chapitre 2 et les deux programmes en résultant ont été appliqués au barrage de l'Oued el Makhazine sur le Loukkos au Maroc. L'auscultation de ce barrage pendant et après la réalisation a fourni des résultats suffisants et fiables. $\mathrm{Si}$ ce barrage a été choisi, c'est que des pressions interstitielles négatives importantes se sont manifestées dans le noyau. Il est donc particulièrement intéressant de vérifier si la théorie est capable, dans un cas similaire, de prédire ces pressions négatives sur la base des caractéristiques des matériaux, résumées dans la table $n^{\circ} 2$. La géométrie du barrage est donnée à la figure 11 .

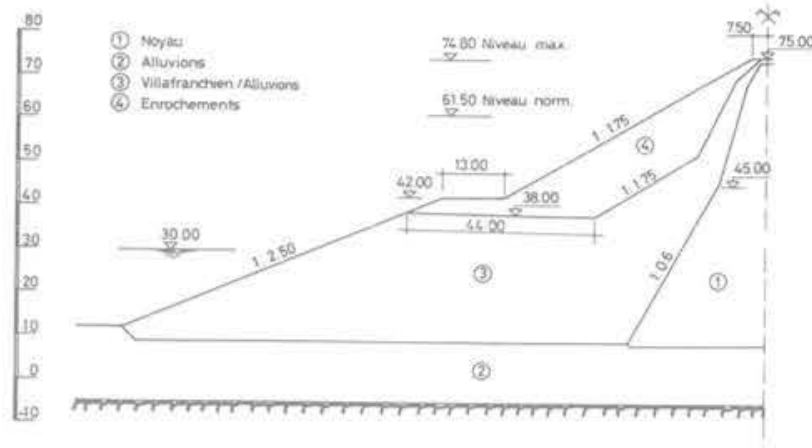

Fig. 11. - Géométrie du barrage et matériaux utilisés.

Le calcul est fait en six étapes simulant ainsi les phases de construction. A chaque étape, la charge appliquée aux nœuds des éléments correspond au poids propre du volume de terre représenté par ces éléments, ce qui donne l'augmentation de la pression interstitielle. Ensuite, la pression interstitielle se dissipe juqu'à l'étape suivante. En répétant le calcul, il est ainsi tenu compte de l'évolution des contraintes et des pressions interstitielles.

Les tassements et les pressions interstitielles sont donnés dans les figures 12,13 et 14 . La figure 12 montre que les tassements calculés concordent avec les mesures effectuées sur l'ouvrage réel. En tenant compte des variations possibles des caractéristiques des matériaux on constate également une bonne appréciation des pressions interstitielles reportées sur la figure 13. La figure 14, finalement, résume les déplacements calculés pour l'état de fin de construction.

\section{CONCLUSIONS}

Le comportement d'un sol non saturé est influencé d'une manière décisive par son degré de saturation. En particulier la succion matricielle joue un rôle important sur l'évolution des pressions interstitielles, ces dernières étant considérées séparément pour l'eau et l'air.

La théorie développée et les programmes en résultant permettent l'introduction de la saturation et des caractéristiques mécaniques et hydrauliques qui en dépendent. La combinaison des calculs pour les déformations instantanées, respectivement différées, permet de simuler fidèlement la construction d'un barrage en matéraux meubles.

L'exemple d'un barrage réalisé montre la comparaison des valeurs calculées avec celles observées et met en évidence le bien-fondé du développement théorique. Le fait que le calcul fasse apparaître les pressions interstitielles négatives effectivement mesurées dans le noyau de ce barrage est d'un intérêt particulier. La présentation d'une méthode simplifiée permet, pour le cas unidimensionel, une bonne appréciation des déformations et pressions interstitielles sans avoir à recourir aux programmes sur ordinateurs. 


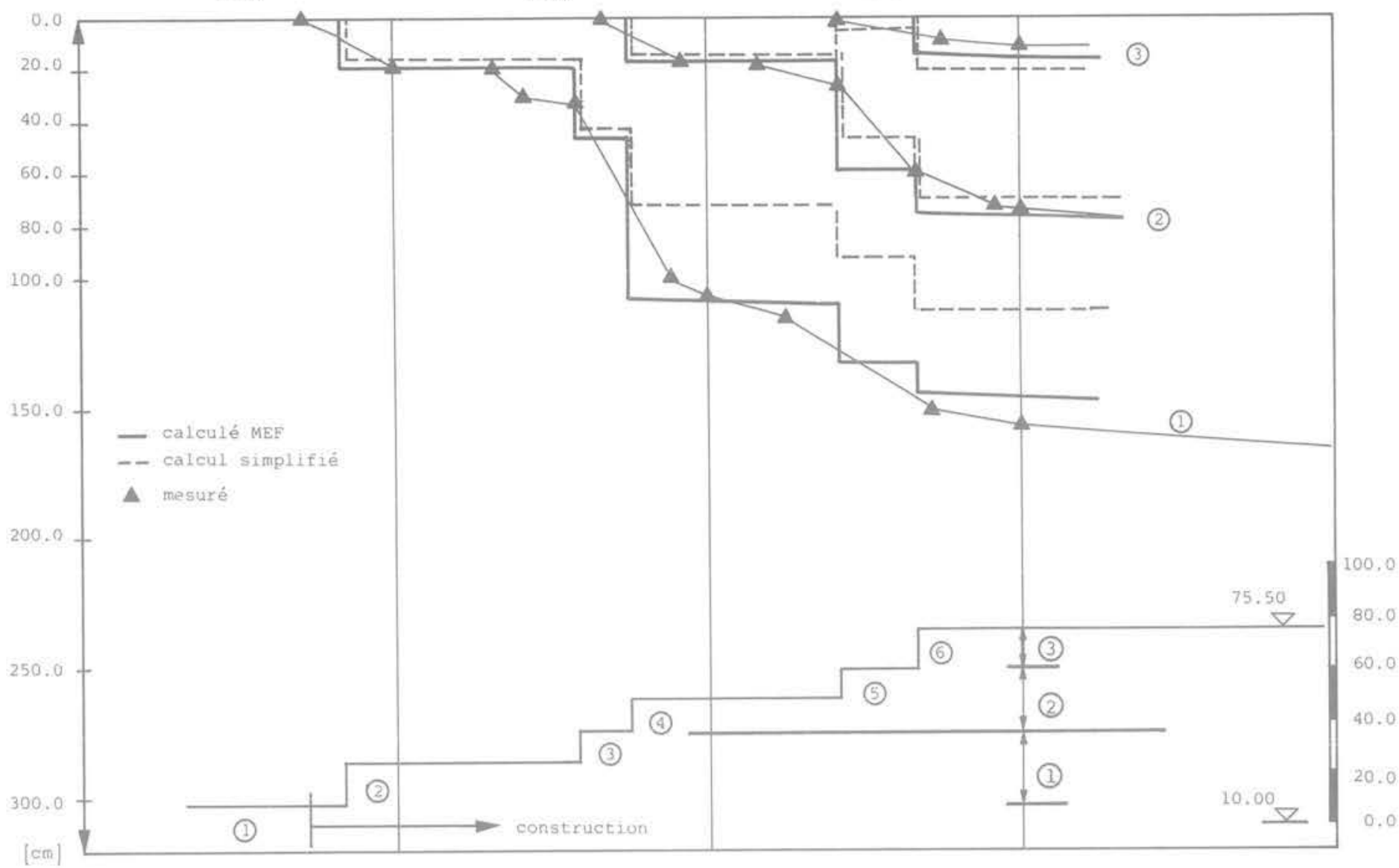

Fig. 12. - Tassements calculés et mesures de trois couches.

1976

1977

1978

1979

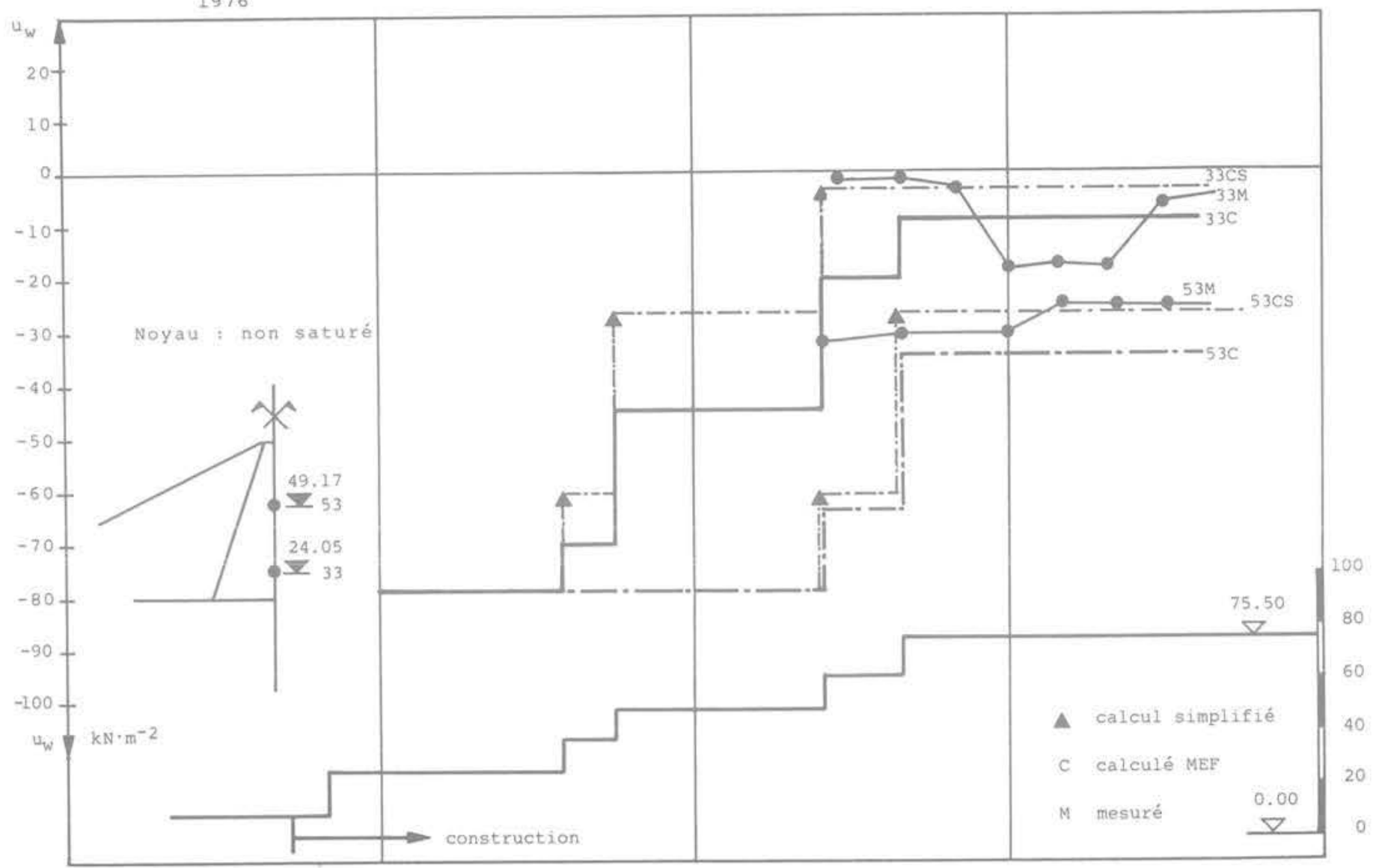

Fig. 13. - Pressions interstitielles calculées et mesurées aux points 33 et 53. 


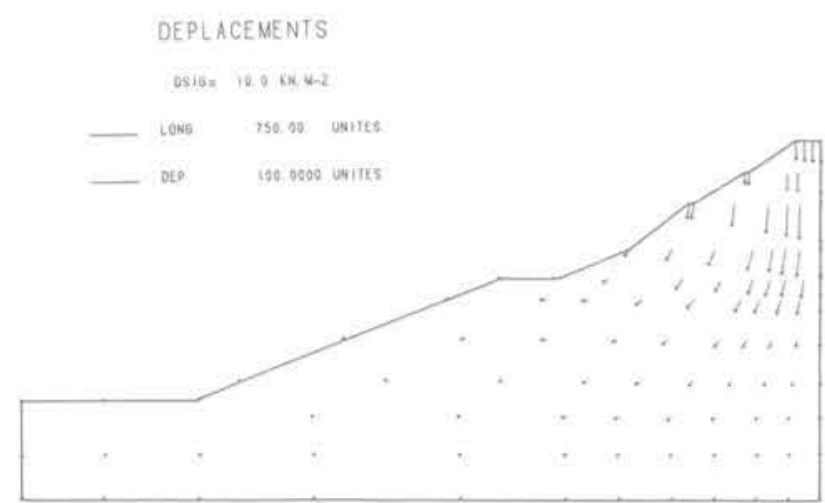

Fig. 14. - Direction des déplacements du barrage à la fin de la construction.

\section{REMERCIEMENTS}

Les auteurs tiennent à remercier l'Administration de l'Hydraulique du Royaume du Maroc à Rabat-Chellah qui leur a permis d'utiliser les mesures d'auscultation du barrage Ouest el Makhazine, Les remerciements vont également à Electrowatt Ingénieurs-Conseils S.A. Maroc qui a mis à disposition les documents y relatifs.

\section{BIBLIOGRAPHIE}

1. EISENSTEIN, Z.; KRISHNAYYA, A.G.; LAW, S.T. (1978). - Analysis of consolidation in cores and earth dams. Num. Methods in Geomech. Ed. Desa, New York, Vol. II, p. 1089-1105.

2. GHABOUSSI, J.; KIM, J.K. (1982). - Analysis of saturated and partially saturated soils. Int. Symposium on Num. Model in Geomech., Zurich, 13-17 sept. 1982 , p. $377-390$.

3. HENKEL, D.J. (1956). - The effect of overconsolidation in the behaviour of clays during shear. Geotechnique 4, p. 139-150.

4. BISHOP, A.W.; BLIGHT, G.E. (1963). - Some aspects of effective stress in saturated and partially saturated soils. Geotechnique 13, p. 177-197.

5. SEKER, E. (1983). - Etude de la déformation d'un massif de sol non saturé. Thèse E.P.F. Lausanne, $\mathrm{n}^{\circ} 492$.

6. VACHAUD, G. (1969). - Analyse des profils hydriques et détermination de $k(\theta)$. Bulletin du B.R.G.M., n4, p. $101-105$.

7. DINIA, N.; SINNIGER, R. (1982). - Non homogenous Rockfill testing and treatment. $14^{e}$ congrès des Grands Barrages, Rio de Janeiro, p. 11-21.

8. TERZAGHI, K. (1951). - Mécanique théorique des sols, Dunod, Paris.

9. JENNING, J.E. : BURLAND, J.B. (1962) - Limitations to the use of effective stresses in partly saturated soils. Géotechnique 12, p. 125-144.

10. ALPAN, I. (1961). - The dissipation function for unsaturated soils. $5^{e}$ Congrès International de Méc. des Sols et des Travaux de Fondation, Paris, vol. 1, p. 3-5.

Liste des symboles

\section{Symboles Unités Définitions}

A

$A_{f}$

a

B

$\stackrel{c}{\mathrm{C}}$

$\mathrm{C}_{\mathrm{c}}$

$c_{1}$

c

[D]

E

$\{\mathrm{F}\}$

$\mathrm{H}$

$h_{a, w}$

Ip

K

[K]

$\mathrm{k}_{\mathrm{a}, \mathrm{w}}$

$\mathrm{m}_{\mathrm{a}}$

$\mathrm{m}_{\mathrm{v}}$

$\mathrm{m}_{\mathrm{ic}}$

$\mathrm{m}_{\mathrm{w}}$

$\mathrm{m}_{0,1}$

OCR

p

$\mathrm{pF}_{\mathrm{c}}$

S

$\mathrm{S}_{\mathrm{r}}$

$S_{r} \propto$

$T_{y}$

U

[U]

$u_{a, w}$

u

$\{\mathrm{V}\}$

w

$w_{L}$

$W_{p}$

$\alpha$

$\triangle$

(8\}

$\{\varepsilon\}$

$\varepsilon_{\nu}$

$\phi$

$\gamma$

$\rho$

$\{\sigma\}$

$\sigma_{c}$

$\chi$

$\chi_{1,2}$

$\psi$

$\psi_{0,1,2}$

$\mathrm{v}$
$\tau$
- Paramètres de la pression interstitielle

- Paramètres de la pression à la rupture

- Indice désignant l'air

- Paramètre de la pression interstitielle

$\mathrm{m}^{-1}$ Matrice de déplacements

$\mathrm{kN} . \mathrm{m}^{-2}$ Cohésion effective

- Indice de compression

$\%$ Coefficient de correction

- Constante d'intégration

$\mathrm{m}^{2} \cdot \mathrm{s}^{-1}$ Coefficient de consolidation

$\mathrm{kN} . \mathrm{m}^{-2}$ Matrice d'élasticité

$\mathrm{kN} . \mathrm{m}^{-2}$ Module d'élasticité

- Indice de vide

kN Forces extérieures

- Coefficient de Henry

m Charge hydraulique

$\%$ Indice de plasticité

$\mathrm{kN} . \mathrm{m}^{-2}$ Module de déformation volumique

$\mathrm{kN} \cdot \mathrm{m}^{-2}$ Matrice de rigidité

$\mathrm{m} \cdot \mathrm{s}^{-1}$ Coefficient de perméabilité (à l'air, à l'eau)

$\%$ Paramètre de compressibilité

$\mathrm{m}^{2} \cdot \mathrm{kN}^{-1}$ Coefficient de compressibilité volumique

$\mathrm{m}^{2} \cdot \mathrm{kN}^{-1}$ Coefficient de compressibilité du squelette

$\mathrm{m}^{2} \cdot \mathrm{kN}^{-1}$ Coefficient de compressibilité du fluide

- Constantes du modèle cedométrique

\% Porosité

- Degré de surconsolidation

$\mathrm{kN} . \mathrm{m}^{-2}$ Pression interstitielle totale

- $\log _{10}$ de la succion

- pF de la frange capillaire

$\%$ Capacité spécifique de saturation

$\%$ Degré de saturation

$\%$ Degré de saturation d'occlusion

s Temps

- Facteur de temps

$\%$ Degré de consolidation

- Matrice de pression non drainée

$\mathrm{kN} \cdot \mathrm{m}^{-2}$ Pressions interstitielles (air, eau)

- Indice désignant le cas non drainé

$\mathrm{m}^{3}$ Volume

$\mathrm{m} \cdot \mathrm{s}^{-1}$ Vitesse

- Indice désignant l'eau

$\%$ Limite de liquidité

$\% \quad$ Limite de plasticité

- Constante de perméabilité

- Variation ou incrément

- Laplacien

m Déplacements

\% Déformations

\% Déformation volumique

degrés. Angle de frottement interne

$\mathrm{kN} . \mathrm{m}^{-3}$ Poids volumique

$\mathrm{tm}^{-3}$ Masse volumique

$\mathrm{kN} . \mathrm{m}^{-2}$ Contraintes

$\mathrm{kN} \cdot \mathrm{m}^{-2}$ Contrainte de préconsolidation

$\%$ Coefficient de Bishop

- Constantes du coefficient de Bishop

$\mathrm{kN} . \mathrm{m}^{-2}$ Succion matricielle

- Constantes de pF

- Coefficient de Poisson

$\mathrm{kN} . \mathrm{m}^{-2}$ Contrainte de cisaillement 
Table 1. - Estimation des caractéristiques et paramètres utilisés.

\begin{tabular}{|c|c|c|c|c|}
\hline Caractéristiques & Symbole & Unité & Valeur approchée & Forme exacte \\
\hline \multirow{4}{*}{ physique } & $w_{L}$ & $\%$ & donnée & \multirow{8}{*}{$\begin{aligned} p F_{c} & =\psi_{0}\left(1-2 \psi_{1}\right) \\
m & =-4 \psi_{0} \psi_{1} \\
\psi & =\gamma_{w} 10^{p} \\
\psi & =\left(u_{a}-u_{w}\right) \\
p F & =\psi_{0}\left(\frac{1-S_{r}}{S_{r}}\right)^{\psi_{1}}\end{aligned}$} \\
\hline & $I_{p}$ & $\%$ & donnée & \\
\hline & $c^{\prime}$ & $\mathrm{kN} \mathrm{m} \mathrm{m}^{-2}$ & $\gamma_{w} 10^{p F_{c}-2} \operatorname{tg} \phi^{\prime}$ & \\
\hline & $\phi^{\prime}$ & $\circ$ & $\sin \phi^{\prime}=\frac{20}{30+I_{p}}$ & \\
\hline \multirow{4}{*}{ succion } & $\mathrm{pF}$ & - & \multirow{2}{*}{$\begin{array}{c}3.75 \sqrt{\frac{I_{p}}{W_{L}}} \\
\begin{array}{r}I_{p}>25=m 6.26-0.046 I_{p} \\
I_{p}<25 m=10^{-3} I_{p}{ }^{2}\left[2.50-0.233 I_{p}\right]+ \\
+0.298 I_{p}\end{array}\end{array}$} & \\
\hline & m. & - & & \\
\hline & $\psi_{0}$ & - & $\frac{2 p F_{c}+m}{2}$ & \\
\hline & $\psi_{1}$ & - & $\frac{m}{2(2 p F c+m)}$ & \\
\hline perméabilité & $\begin{array}{l}\mathrm{kw} \\
\mathrm{n}_{1}\end{array}$ & $m \cdot s^{-1}$ & $\begin{array}{l}2.7 \times 10^{-2} \psi_{0} \\
2 \log \psi_{0}+3\end{array}$ & $k_{w r}=\left(\frac{s_{r}-s_{r} \min }{1-s_{r} \min }\right)^{n_{1}}$ \\
\hline \multirow{4}{*}{ déformabilité } & mo & $\mathrm{kN} \cdot \mathrm{m}^{-2}$ & $2.3\left(1+2.7 \frac{W_{1}}{100}\right)$ & \multirow{4}{*}{$E_{\text {oed }}=m o\left(\frac{\sigma^{\prime}}{p a}\right)^{m_{1}}$} \\
\hline & $m_{1}$ & & $1+\log \left(1-\frac{2.3}{\mathrm{mo}}\right)$ & \\
\hline & $\mathrm{c}_{\mathrm{c}}$ & - & $0,007\left(W_{L}-10\right)$ & \\
\hline & $v$ & & $\frac{10+I_{p}}{40+I_{p}}$ & \\
\hline \multirow[t]{2}{*}{ coefficient } & $x_{1}$ & - & 0.25 & \multirow{2}{*}{$x=\left(\frac{1-x_{1}-x_{1}}{x_{1}}\right)$} \\
\hline & $x_{2}$ & - & $2 \log \psi_{0}+1$ & \\
\hline
\end{tabular}


Table 2. - Caractéristiques des sols.

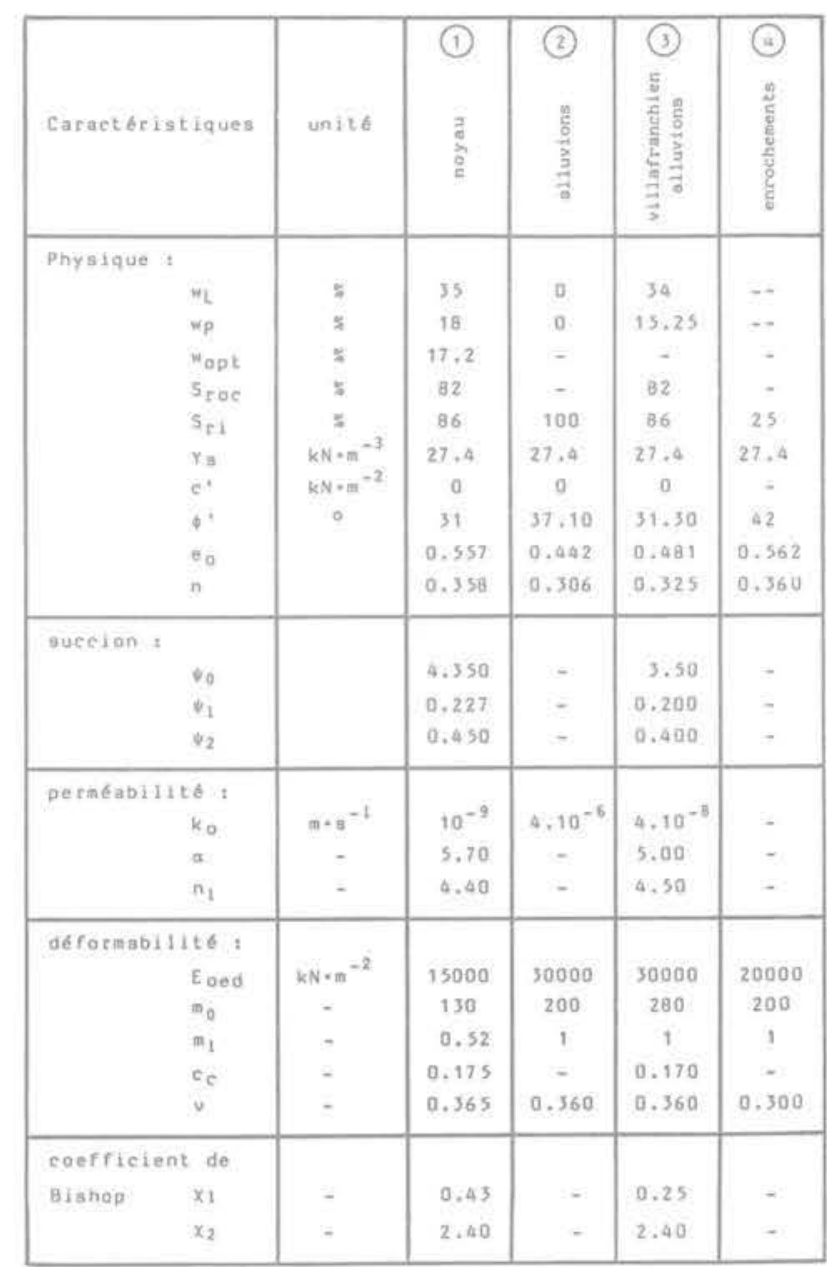




\section{ANNEXE}

$\mathrm{S}_{\mathrm{r}}=0.8 \mathrm{~s}$

\begin{tabular}{|c|c|c|c|c|c|c|c|c|c|c|}
\hline \multicolumn{9}{|c|}{$\varepsilon_{V}=0.010$} & \multicolumn{1}{c|}{$E_{\mathrm{V}}=0.025$} \\
\hline$W_{\mathrm{L}}$ & 5 & 10 & 15 & 20 & 30 & 5 & 10 & 15 & 20 & 30 \\
\hline 10. & 20.000 & 5.482 & 3.229 & - & - & 20.000 & 3.270 & 1.866 & - & - \\
20. & 18.034 & 4.479 & 2.718 & 2.168 & - & 16.314 & 2.781 & 1.665 & 1.322 & - \\
30. & 13.687 & 3.948 & 2.422 & 1.939 & 2.328 & 10.880 & 2.523 & 1.540 & 1.229 & 1.411 \\
40. & 11.610 & 3.598 & 2.221 & 1.783 & 2.154 & 8.852 & 2.352 & 1.451 & 1.163 & 1.342 \\
50. & 10.334 & 3.343 & 2.072 & 1.665 & 2.022 & 7.741 & 2.227 & 1.384 & 1.111 & 1.287 \\
60. & - & - & 1.956 & 1.574 & 1.916 & - & - & 1.330 & 1.070 & 1.242 \\
70. & - & - & - & 1.500 & 1.829 & - & - & - & 1.035 & 1.204 \\
30. & - & - & - & - & 1.755 & - & - & - & - & 1.171 \\
\hline
\end{tabular}

$\mathrm{S}_{\mathrm{r}}=0.90$

\begin{tabular}{|c|c|c|c|c|c|c|c|c|c|c|}
\hline \multicolumn{9}{|c|}{$\varepsilon_{v}=0.010$} & \multicolumn{9}{c|}{$\varepsilon_{v}=0.025$} \\
\hline If & 5 & 10 & 15 & 20 & 30 & 5 & 10 & 15 & 20 & 30 \\
\hline 10. & 20.000 & 4.664 & 2.758 & - & - & 20.000 & 2.782 & 1.594 & - & - \\
20. & 15.349 & 3.850 & 2.347 & 1.875 & - & 13.885 & 2.390 & 1.438 & 1.143 & - \\
30. & 11.717 & 3.416 & 2.106 & 1.688 & 2.010 & 9.314 & 2.183 & 1.339 & 1.070 & 1.218 \\
40. & 9.983 & 3.128 & 1.941 & 1.559 & 1.869 & 7.611 & 2.045 & 1.268 & 1.017 & 1.164 \\
50. & 8.918 & 2.918 & 1.818 & 1.463 & 1.761 & 6.681 & 1.944 & 1.214 & 0.976 & 1.121 \\
60. & - & - & 1.722 & 1.387 & 1.674 & - & - & 1.171 & 0.943 & 1.085 \\
70. & - & - & - & 1.326 & 1.602 & - & - & - & 0.915 & 1.455 \\
80. & - & - & - & - & 1.542 & - & - & - & - & 1.028 \\
\hline
\end{tabular}

$S_{T}=0.95$

\begin{tabular}{|c|c|c|c|c|c|c|c|c|c|c|}
\hline \multicolumn{2}{|c|}{$\varepsilon_{V}=0.010$} & \multicolumn{1}{c|}{$\varepsilon_{V}=0.025$} \\
\hline I. & 5 & 10 & 15 & 20 & 30 & 5 & 10 & 15 & 20 & 30 \\
\hline 10. & 20.000 & 3.200 & 1.905 & - & - & 20.000 & 1.909 & 1.101 & - & - \\
20. & 10.538 & 2.686 & 1.651 & 1.321 & - & 9.533 & 1.668 & 1.011 & 0.805 & - \\
30. & 8.122 & 2.410 & 1.498 & 1.204 & 1.413 & 6.457 & 1.540 & 0.953 & 0.763 & 0.857 \\
40. & 6.972 & 2.225 & 1.393 & 1.121 & 1.325 & 5.315 & 1.455 & 0.910 & 0.732 & 0.825 \\
50. & 6.266 & 2.090 & 1.314 & 1.059 & 1.256 & 4.694 & 1.392 & 0.877 & 0.707 & 0.800 \\
60. & - & - & 1.251 & 1.010 & 1.201 & - & - & 0.851 & 0.687 & 0.778 \\
70. & - & - & - & 0.970 & 1.155 & - & - & - & 0.670 & 0.760 \\
80. & - & - & - & - & 1.116 & - & - & - & - & 0.744 \\
\hline
\end{tabular}

